\title{
Proteoglycan-4 is an essential regulator of synovial macrophage polarization and inflammatory macrophage joint infiltration
}

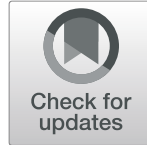

Marwa Qadri ${ }^{1}$, Gregory D. Jay ${ }^{2}$, Ling X. Zhang ${ }^{2}$, Tannin A. Schmidtt ${ }^{3}$ Jennifer Totonchy ${ }^{4}$ and Khaled A. Elsaid ${ }^{4^{*}}$ (D)

\begin{abstract}
Background: Synovial macrophages perform a multitude of functions that include clearance of cell debris and foreign bodies, tissue immune surveillance, and resolution of inflammation. The functional diversity of macrophages is enabled by distinct subpopulations that express unique surface markers. Proteoglycan-4 (PRG4) is an important regulator of synovial hyperplasia and fibrotic remodeling, and the involvement of macrophages in PRG4's synovial role is yet to be defined. Our objectives were to study the PRG4's importance to macrophage homeostatic regulation in the synovium and infiltration of pro-inflammatory macrophages in acute synovitis and investigate whether macrophages mediated synovial fibrosis in Prg4 gene-trap (Prg $4^{G T / G T}$ ) murine knee joints.
\end{abstract}

Methods: Macrophage phenotyping in $\mathrm{Prg} 4^{G T / G T}$ and $\operatorname{Prg} 4^{+/+}$joints was performed by flow cytometry using panmacrophage markers, e.g., CD11b, F4/80, and surface markers of M1 macrophages (CD86) and M2 macrophages (CD206). Characterizations of the various macrophage subpopulations were performed in 2- and 6-month-old animals. The expression of inflammatory markers, IL-6, and iNOS in macrophages that are CD86+ and/or CD206+ was studied. The impact of Prg4 recombination on synovial macrophage populations of 2- and 6-month-old animals and infiltration of pro-inflammatory macrophages in response to a TLR2 agonist challenge was determined. Macrophages were depleted using liposomal clodronate and synovial membrane thickness, and the expression of fibrotic markers a-SMA, PLOD2, and collagen type I (COL-I) was assessed using immunohistochemistry.

Results: Total macrophages in $\operatorname{Prg} 4^{G T / G T}$ joints were higher than $\operatorname{Prg} 4^{+/+}$joints $(p<0.0001)$ at 2 and 6 months, and the percentages of CD86+/CD206- and CD86+/CD206+ macrophages increased in Prg $4^{G T / G T}$ joints at 6 months ( $p<$ 0.0001), whereas the percentage of CD86-/CD206+ macrophages decreased $(p<0.001)$. CD86+/CD206- and CD86+/ CD206+ macrophages expressed iNOS and IL-6 compared to CD86-/CD206+ macrophages $(p<0.0001)$. Prg 4 reexpression limited the accumulation of CD86+ macrophages $(p<0.05)$ and increased CD86-/CD206+ macrophages $(p<0.001)$ at 6 months. Prg4 recombination attenuated synovial recruitment of pro-inflammatory macrophages in 2month-old animals $(p<0.001)$. Clodronate-mediated macrophage depletion reduced synovial hyperplasia, a-SMA, PLOD2, and COL-I expressions in the synovium ( $p<0.0001)$.

Conclusions: PRG4 regulates the accumulation and homeostatic balance of macrophages in the synovium. In its absence, the synovium becomes populated with M1 macrophages. Furthermore, macrophages exert an effector role in synovial fibrosis in $\operatorname{Prg} 4^{G T / G T}$ animals.

Keywords: PRG4, Synovial macrophages, Bone-marrow-derived macrophages, Synovial hyperplasia

\footnotetext{
* Correspondence: elsaid@chapman.edu

${ }^{4}$ Department of Biomedical and Pharmaceutical Sciences, Chapman

University School of Pharmacy, Rinker Health Sciences Campus, 9401

Jeronimo Road, Irvine, CA 92618, USA

Full list of author information is available at the end of the article
}

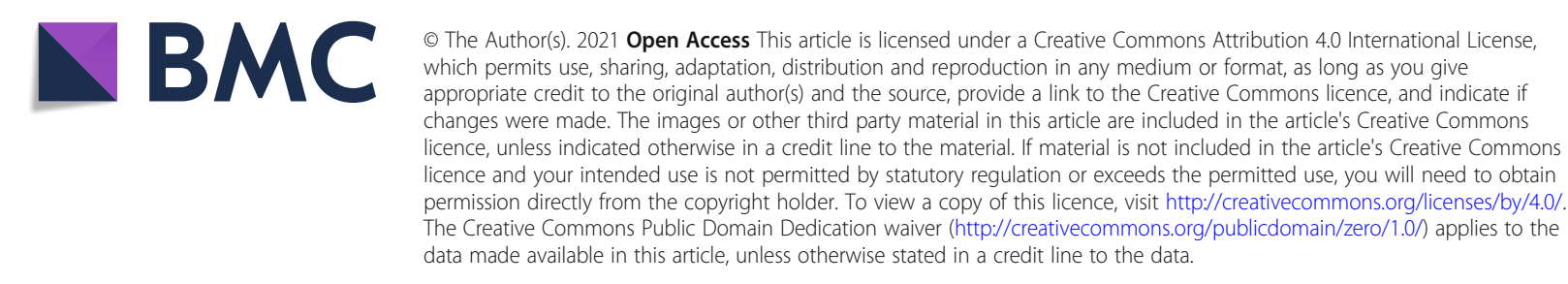




\section{Background}

The synovium is a soft tissue comprised of a surface layer, the intima, and an underlying subintima $[1,2]$. The synovial intima is 1-3-cell-layer thick, with two cell types: fibroblast-like synoviocytes and macrophages [1, 2]. The subintima is less cellular with significant collagen type I content, microvascular blood, and lymph vessels [2]. Synovial macrophages comprise distinct subsets of heterogenous cell populations that perform various functions including clearance of cell debris and foreign bodies, tissue immune surveillance, and resolution of inflammation [3-5]. The synovial microenvironment regulates macrophage heterogeneity as it controls the polarization of macrophages on a spectrum of proinflammatory and anti-inflammatory functions [6-9]. At one end of the spectrum is the M1 macrophage phenotype, with a pro-inflammatory role mediated by the production of high levels of interleukin-1 beta (IL-1 $\beta$ ), tumor necrosis factor alpha (TNF- $\alpha$ ), IL-6, inducible nitric oxide synthase (iNOS) expression specifically in murine macrophages, and generation of reactive oxygen species [10-12]. At the opposite end is the M2 macrophage phenotype, which is characterized by the production of anti-inflammatory cytokines; IL-10, interleukin-1 receptor antagonist (IL-1Ra), and transforming growth factor beta (TGF- $\beta$ ) $[9,10,13]$. M1 macrophages have been associated with the exacerbation of synovial inflammation, and related joint destruction in OA and RA [12, 14], while the population of the synovium by M2 macrophages was associated with resolution of joint inflammation [15].

Proteoglycan-4 (PRG4) is a mucinous glycoprotein secreted by fibroblast-like synoviocytes and superficial zone chondrocytes $[16,17]$. PRG4 functions as a boundary lubricant between apposed cartilage surfaces, which prevents mitochondrial dysregulation and superficial zone chondrocyte apoptosis [18]. Biologically, PRG4 engages a number of receptors including $\mathrm{CD} 44$ and the Toll-like receptors 2 and 4 (TLR2 and TLR4) [19-21]. PRG4-CD44 interaction inhibits nuclear factor kappa B (NF-KB) nuclear translocation in human synovial fibroblasts and attenuates IL-1 $\beta$-induced synoviocyte proliferation and expression of matrix-degrading enzymes [22]. PRG4 regulates synovial fibroblast to myofibroblast transition and reduces the expression of fibrotic markers: alpha smooth muscle actin ( $\alpha$-SMA) and collagen type I $[23,24]$. PRG4 also suppresses the activation of TLR2 and TLR4 receptors by DAMPs in OA synovial fluid aspirates [21]. Synovial hyperplasia and fibrosis, that progressed with age, are evident in murine knee joints lacking Prg4 expression [23, 25, 26]. The biological role of PRG4 helps explain its disease-modifying activity in rodent and pig models of posttraumatic osteoarthritis (PTOA), where PRG4 reduced cartilage degeneration and enhanced its repair [27, 28]. While PRG4's effects on synovial fibroblasts have been characterized, its contribution to synovial macrophage polarization on the M1-M2 spectrum remains largely unknown. Preliminary evidence suggests that PRG4 may influence macrophage responsiveness as peritoneal macrophages from $\operatorname{Prg} 4$ knockout mice secreted higher levels of IL- $1 \beta$ following TLR stimulation compared to peritoneal macrophages from Prg4 expressing mice [29].

Synovitis is a significant and common finding in patients with knee OA and is an independent predictor of radiographic OA progression [30]. Synovitis is characterized by synovial hyperplasia, subintimal fibrosis, and neovascularization [31]. Macrophages and T cells are the predominant immune cells encountered in the OA synovium, and most inflammatory cytokines produced in synovitis are attributed to activated synovial macrophages [31]. Synovial fibrosis is a maladaptive resolving response to chronic synovitis and is characterized by increased cross-linked collagen I deposition, due to a combination of enhanced collagen production and PLOD2-mediated crosslinking, and transition of synovial fibroblasts to activated myofibroblasts, characterized by de novo $\alpha$-SMA expression [23, 24]. Synovial fibrosis has an immune-mediated etiology, and it is hypothesized that DAMPs trigger TLR-mediated activation of synovial macrophages and subsequent release of TGF- $\beta$ which in turn drives the fibrotic remodeling of the synovium [32].

In this study, we aimed to investigate the role of PRG4 in regulating macrophage phenotype polarization and accumulation in synovial tissues and the recruitment of pro-inflammatory macrophages in response to acute synovitis, and to evaluate whether chronic synovitis and synovial fibrosis in Prg4 null joints are mediated by an enhanced polarization towards a pro-inflammatory macrophage phenotype. In the course of our investigation, we studied synovium-resident macrophages (SRMs) and total macrophages in isolated synovial tissues using differential and overlapping markers. We also investigated the recruitment of pro-inflammatory macrophages that have originated from circulating monocytes in response to a TLR2 agonist intra-articular challenge. We hypothesized that PRG4 maintains synovial macrophage homeostasis, and in its absence, macrophages shift towards an M1 pro-inflammatory phenotype resulting in chronic synovitis and fibrotic remodeling.

\section{Methods}

\section{Animals and study overview}

The Prg4 gene-trap (Prg4 ${ }^{G T}$ ) mouse (stock no. 025740, JAX, USA) is born lacking Prg4 expression which can be restored via CRE-mediated recombination [26]. The $\operatorname{Prg}^{G T / G T}$ animal displays significant synovial hyperplasia and subintimal fibrosis and recombination appeared to 
attenuate these changes [23]. In this study, Prg4 recombination $\left(\operatorname{Prg} 4^{G T R / G T R}\right)$ occurred in 3-week-old animals via intraperitoneal injection of tamoxifen $(0.1 \mathrm{mg} / \mathrm{g}$ in $100 \mu \mathrm{L}$ corn oil vehicle) daily for 10 days, using vehicleadministered gene-trap animals as controls. We have also used $\operatorname{Prg} 4^{+/+}$animals (stock \# 101045, JAX; B6/129S background). Animals were studied at 2 or 6 months of age and depletion of macrophages was performed when animals were 4-month-old, and we included randomly assigned litter and age-matched males and females in our experimental groups. The number of animals per group was based on our previous analysis of the effect of Prg4 recombination on synovial fibrosis using alphasmooth muscle actin ( $\alpha$-SMA) staining as our primary endpoint [23]. Our studies were approved by the IACUC committee at Chapman University. All experiments were performed according to established guidelines and regulations.

We studied SRMs in 2- and 6-month-old $\operatorname{Prg} 4^{\text {GT/GT }}$ and $\operatorname{Prg} 4^{+/+}$knee joints. We identified SRMs using CD68-a tissue-resident macrophage marker-CD11b, and F4/80 [5, 14]. SRMs were identified as CD68+ $\mathrm{CD} 11 \mathrm{~b}+\mathrm{F} 4 / 80+\mathrm{MHC}$ class II-. We also studied the expression of CX3CR1 receptor in SRMs of $\operatorname{Prg} 4^{G T / G T}$ and $\operatorname{Prg}^{+/+}$joints as CX3CR1+ SRMs were recently shown to play an anti-inflammatory homeostatic role [5]. Total macrophages in the synovium were identified as $\mathrm{CD} 11 \mathrm{~b}+\mathrm{F} 4 / 80+\mathrm{MHC}$ class II- and a combination of either CD86+/CD206-, CD86+/CD206+ or CD86 -/CD206+. In characterizing these macrophages, we utilized CD86 (an M1 marker) [33] and CD206 (an M2 marker) [34] to provide us with an understanding of the balance between the M1 and M2 phenotypes under different experimental conditions. We confirmed the proinflammatory phenotype of CD86+ macrophages by iNOS and IL-6 staining, and the anti-inflammatory phenotype of M2 macrophages by arginase-1 (Arg-1) staining [35]. We also studied the impact of Prg4 recombination on total macrophage numbers and the balance between CD86+ and CD206+ macrophages. To further characterize the role of PRG4 in regulating the recruitment of pro-inflammatory macrophages, we induced synovitis in $\operatorname{Prg} 4^{G T / G T}$ and $\operatorname{Prg} 4^{\text {GTR/GTR }}$ knee joints using Pam3CSK4 (Invivogen, USA), a TLR2 agonist [36]. We identified newly infiltrated pro-inflammatory macrophages, based on MHC class II and Ly-6C positivity as CD11b+ F4/80+ MHC class II+ Ly-6C+CD86+ $[14,37]$. We also depleted macrophages in $\operatorname{Prg} 4^{G T / G T}$ joints using intra-articular (IA) clodronate liposomes and confirmed macrophage depletion by $\mathrm{CD} 11 \mathrm{~b}$ immunoprobing in the synovial tissues of these animals. We selected an intermediate endpoint, day 15 following clodronate treatment initiation, to evaluate changes to CD86+ and/or CD206+ macrophages in synovial tissues. Using immunohistochemistry, we also studied the impact of macrophage depletion at day 30 on synovial hyperplasia and fibrosis in $\operatorname{Prg} 4^{G T / G T}$ joints. Finally, we supplemented our in vivo studies with in vitro studies using murine M2a BMDMs. Our rationale to study M2a macrophages was based on the current understanding that M2 macrophages are the predominant population in the synovium in the absence of an inflammatory stimulus. We used an established differentiation protocol to generate M2a BMDMs and tested whether rhPRG4 treatment modulated the secretion of cytokines and chemokines in TLR2 agonist-stimulated M2a BMDMs. We focused our efforts on the M2a subtype given its role in resolving inflammation and promoting wound healing $[9,10]$. An overview of the experimental approach for our in vivo studies is shown in Supplementary Figure 1.

\section{Immunophenotyping of various macrophage populations} Joint capsular tissues were isolated, pooled from two knee joints to generate an independent sample, and digested using collagenase-D $(2 \mathrm{mg} / \mathrm{mL})$ (Sigma Aldrich, USA) $+1 \mu \mathrm{L}$ RNase-free DNase I $(1 \mathrm{U} / \mu \mathrm{L})$ (ThermoFisher Scientific, USA) in Hanks' balanced salt solution $(1 \mathrm{~mL})$ for $1 \mathrm{~h}$ at $37^{\circ} \mathrm{C}$. Cell suspensions were then filtered through a 40- $\mu \mathrm{m}$ nylon mesh (Sigma Aldrich), and cells were pelleted at $3000 \mathrm{rpm}$ for $5 \mathrm{~min}$. Cells were stained with a viability dye (Zombie Violet; BioLegend, USA) and fluorochrome-conjugated antibodies as described below. The numbers of macrophages of interest were estimated using Precision Counting beads (BioLegend). Our panel was designed using the BD Horizon Guided Panel Solution tool, and the positivity threshold for marker staining was determined using fluorescence minus one (FMO) panel controls. Fluorochromeconjugated antibodies included Alexa Fluor 488-antiiNOS and PE-anti-Arg-1 (ThermoFisher Scientific), APC-Cy7-anti-CD11b, FITC-anti-Ly-6C, PE-Cy7 antiCD86 and PerCP-Cy5.5 anti-I-A/I-E (MHC class II) (BD Biosciences, USA), PE-anti-CD68, Brilliant Violet 510anti-F4/80, Alexa fluor 488-anti-CX3CR1, PE-anti-IL-6, and APC-anti-CD206 (BioLegend) and were used at recommended dilutions. Cells were suspended in FACS blocking buffer $(0.5 \%$ BSA $+2 \%$ FBS in PBS) for $10 \mathrm{~min}$ on ice and then incubated with the antibody mix in FACS staining buffer $(0.5 \%$ BSA $+0.05 \%$ sodium azide in PBS) for $20 \mathrm{~min}$ on ice. Intracellular staining of Arg1, CD68, IL-6, and iNOS proteins was performed following surface marker staining. Cells were suspended in BD Cytofix/Cytoperm buffer containing $4.2 \%$ formaldehyde with fluorochrome-conjugated antibodies diluted according to manufacturer's recommendations and incubated for $20 \mathrm{~min}$ on ice. Subsequently, cells were washed twice with FACS washing buffer followed by flow 
cytometric analysis (BD FACSAria). Flow cytometry plots and analyses were generated using Flow $\mathrm{Jo}^{\circ}$ software (BD Biosciences). We performed two technical replicates for each independent biological sample, and the analysis output was the average of the two technical replicates.

\section{Generation of M2a BMDMs}

The bone marrows of the femurs and tibias of 2-monthold Prg $4^{G T / G T}$ and $\operatorname{Prg} 4^{+/+}$animals were isolated, and BMDMs were generated as described [36, 38]. On day 7 , M2a BMDMs were induced by IL-4 + IL-13 $(20 \mathrm{ng} / \mathrm{mL}$ for both cytokines; R\&D Systems) for 24h [38].

Target gene expression and analysis of secreted cytokines and chemokines by Prg $4{ }^{\text {GT/GT }}$ BMDMs following TLR2 stimulation \pm rhPRG4

M2a BMDMs from 2-month-old Prg $4^{G T / G T}$ and $P r g 4^{+/+}$ joints $(500,000$ cells per well) were treated with Pam3CSK4 (300ng/mL) or cultured as untreated control for $24 \mathrm{~h}$, in serum-free DMEM/F12 media, followed by RNA isolation, cDNA synthesis, and quantitative PCR as described [36]. The cycle threshold (Ct) values of genes of interest (Nos2; gene symbol for iNOS, IL-1 $\beta, I L-6, I L-$ 10 , and $T G F-\beta$ ) were normalized to the $\mathrm{Ct}$ value of $G A P D H$ in the same sample, and relative expression was calculated using the $2^{-\Delta \Delta \mathrm{Ct}}$ method [39]. The following primers and probes were used: Nos2 (Mm00440502_ m1), IL-1 (Mm00434228_m1), IL-6 (Mm00446190_ $\mathrm{m} 1$ ), IL-10 (Mm01288386_m1), TGF- $\beta$ (Mm01178820_ $\mathrm{m} 1$ ), and GAPDH (Mm99999915_g1) (all available from ThermoFisher Scientific). M2a BMDMs from Prg4 ${ }^{G T / G T}$ animals were treated with Pam3CSK4 \pm rhPRG4 [40] $(200 \mu \mathrm{g} / \mathrm{mL})$ for $24 \mathrm{~h}$ followed by gene expression studies and profiling of secreted cytokines and chemokines using Proteome Profiler Mouse Cytokine Array Kit, Panel A (R\&D Systems). Signal intensities of protein spots were quantified using Image $\mathrm{J}^{\circ}$ and normalized to signal intensities of reference spots. Data are presented as mean \pm S.D. of integrated pixel densities from three independent experiments.

IA administrations of Pam3CSK4, vehicle, rhPRG4, or PBS IA injections were performed in the knee joints of 2month-old mice under inhaled isoflurane gas anesthesia (5\% induction and 2-3\% maintenance). The mouse skin was shaved and cleansed with a topical antiseptic. IA injections were performed under a magnifying lens, and a total of $10 \mu \mathrm{L}$ was delivered through the patellar tendon. The effects of IA Pam3CSK4 $(3 \mu \mathrm{g}$ in $10 \mu \mathrm{L})$ were compared to vehicle (sterile water; $10 \mu \mathrm{L}$ ) in $\operatorname{Prg} 4^{G T / G T}$ and Prg $4^{\text {GTR/GTR }}$ animals. IA rhPRG4 $(1 \mathrm{mg} / \mathrm{mL} ; 10 \mu \mathrm{L})$ or phosphate-buffered saline (PBS) $(10 \mu \mathrm{L})$ injections were performed in $\operatorname{Prg} 4^{G T / G T}$ animals, and analysis of
$\mathrm{CD} 11 \mathrm{~b}+\mathrm{F} 4 / 80+\mathrm{MHC}$ class II- CD86+ iNOS+ and $\mathrm{CD} 11 \mathrm{~b}+\mathrm{F} 4 / 80+\mathrm{MHC}$ class II- CD86+ IL-6+ macrophages was conducted as described above at $24 \mathrm{~h}$.

Macrophage depletion using clodronate liposomes and its impact on synovial fibrosis in Prg $4{ }^{\mathrm{GT} / \mathrm{GT}}$ knee joints $\operatorname{Prg} 4^{G T / G T}$ joints were injected with clodronate liposomes $(10 \mu \mathrm{L} ; 18.4 \mathrm{mM})$ or PBS liposomes $(10 \mu \mathrm{L})$ (Encapsula Nanosciences, USA) twice weekly for 2 weeks, and on day 15 following treatment initiation, we quantified the percentages of $\mathrm{CD} 11 \mathrm{~b}+\mathrm{F} 4 / 80+\mathrm{MHC}$ class II- CD86+ or CD86-/CD206+ macrophages and the number of CD86+ macrophages as described above. IA clodronate or PBS liposomes injections were conducted as described above. In a parallel study, $\operatorname{Prg} 4^{G T / G T}$ joints were treated with clodronate, or PBS liposomes as described above and the joints were harvested on day 30 following treatment initiation, and age-matched $\mathrm{Prg} 4^{+/+}$joints (4 months) were used as controls. The joints were decalcified, paraffin-embedded, and sectioned. Sections were stained with hematoxylin \& eosin $(\mathrm{H} \& \mathrm{E})$, and synovial membrane thickness was determined using the mean of five thickness measurements per specimen. Alternatively, sections were permeabilized with $0.1 \%$ triton $\mathrm{x} 100$ for 10 min followed by blocking with staining buffer for 20 min. Sections were incubated with rabbit polyclonal antibodies against $\alpha$-SMA (Abcam, USA), procollagenlysine-2-oxygultarate-5-dioxygenase 2 (PLOD2) (Ab cam), collagen I (COL-I) (Abcam), and CD11b (Novus, USA) (1:100 dilutions for all antibodies) overnight at $4^{\circ} \mathrm{C}$. Following washing with PBS, sections were incubated with Cy3-goat anti-rabbit IgG at 1:200 dilution for $1 \mathrm{~h}$ at room temperature and imaged using a confocal microscope (Olympus BX 51). Regions of interest in synovia of different animals were defined, and integrated OD $\left(\mathrm{Lum}^{*} \mu \mathrm{m}^{2}\right)$ values were computed and compared across groups.

\section{Statistical analyses}

Statistical analyses were conducted as we previously described [23]. We used unpaired, paired Student's $t$ test, and one-way or two-way ANOVAs followed by Tukey's post hoc tests, with statistical significance set at $p<0.05$. The specific test type is stated in figure legends.

\section{Results}

The tissue-resident fraction of macrophages was reduced in Prg4 $4^{G T / G T}$ synovia, and total macrophages liberated from synovial tissue digestion exhibited an age-dependent shift to a predominantly CD86+ pro-inflammatory phenotype and in vivo rhPRG4 treatment reduced inflammatory markers' expression in this specific macrophage population. 
The study of SRMs was performed according to the scheme presented in Supplementary Figure 1A, and gating of this macrophage population was conducted as shown in Supplementary Figure 2 and Fig. 1A. In addition, the study of total macrophages in the synovial tissues was conducted according to the scheme presented in Supplementary Figure 1A and was identified according to the gating strategy in Supplementary Figure 2 and Fig. 1B. The percentages of total macrophages that were identified as SRMs in 2- and 6-month-old $\operatorname{Prg} 4^{G T / G T}$ joints were lower than age-matched $\operatorname{Prg} 4^{+/+}$ joints $(p<0.001 ; p<0.0001 ;$ Fig. 1C). In addition, 6month-old $\operatorname{Prg} 4^{G T / G T}$ joints had a lower percentage of SRMs compared to 2-month-old Prg4 ${ }^{G T / G T}$ joints ( $p<$ 0.001; Fig. 1C), whereas $\operatorname{Prg} 4^{+/+}$joints did not show a significant alteration in SRM percentage as animals aged $(p>0.05)$. Representative flow cytometry plots showing CX3CR1+ SRMs in $\operatorname{Prg} 4^{+/+}$and $\operatorname{Prg} 4^{G T / G T}$ animals are shown in Fig. 1D. The CX3CR1+ fraction of SRMs declined in $\operatorname{Prg} 4^{G T / G T}$ animals as they aged. While the percentage of CX3CR1+ SRMs in 2-month-old Prg4 ${ }^{G T / G T}$ animals was higher compared to age-matched $\operatorname{Prg} 4^{+/+}$ animals $(p<0.05$; Fig. 1E), CX3CR1+ SRM percentage in 6-month-old Prg4 ${ }^{G T / G T}$ joints was not significantly different from $\operatorname{Prg} 4^{+/+}$joints but was lower than 2-monthold Prg $4^{G T / G T}$ joints $(p<0.01)$. Total macrophages were higher in joints of 2- and 6-month-old Prg4 ${ }^{G T / G T}$ animals compared to $\operatorname{Prg} 4^{+/+}$animals $(p<0.0001$; Fig. 1F). Macrophages in the synovial tissues comprised heterogenous subpopulations with differential expression patterns of CD86 and/or CD206 proteins. CD86+/ CD206- and CD86+/CD206+ macrophages were higher in 2- and 6-month-old $\operatorname{Prg} 4^{G T / G T}$ joints compared to Prg $^{+/+}$animals $(p<0.05$ for all comparisons; Fig. 1G), while CD86-/CD206+ macrophages were depleted in 6month-old $\operatorname{Prg} 4^{G T / G T}$ joints ( $p<0.001$; Fig. 1G). The M1/ M2 ratio increased with age in $P r g 4^{G T / G T}$ animals compared to $\operatorname{Prg} 4^{+/+}$animals (Fig. $1 \mathrm{H}$ ). M1/M2 ratio was higher in 6-month-old Prg4 ${ }^{G T / G T}$ synovia compared to 2-month-old $\mathrm{Prg}_{4}^{+/+}$and 6-month-old $\operatorname{Prg} 4^{+/+}$synovia $(p<0.001 ; p<0.0001$; Fig. $1 \mathrm{H})$. These findings support that as Prg4 $4^{G T / G T}$ animals aged, the total number of macrophages increased, while the fraction of the macrophages that are tissue-resident declined and that change was associated with a shift towards a higher M1/M2 ratio.

CD86+ and/or CD206+ macrophages in 2-month-old Prg4 ${ }^{G T / G T}$ joints were further classified according to their iNOS and IL-6 expression status (Fig. 2A). CD86+/ CD206- and CD86+/CD206+ macrophages contained higher percentages of IL-6+/iNOS+ cells compared to CD86-/CD206+ macrophages, and the magnitude of this increase was $\sim 4.5$-fold $(p<0.0001$; Fig. $2 \mathrm{~B})$. Similarly, mean IL-6 and iNOS staining intensities were higher in CD86+/CD206- and CD86+/CD206+ macrophages compared to corresponding mean intensities in CD86-/CD206+ macrophages ( $p<0.05$; Fig. $2 \mathrm{C}$ ). We further studied CD206+ macrophages to identify whether they co-expressed classical M2 markers, e.g., Arg-1. We observed that in 2-month-old $\operatorname{Prg} 4^{G T / G T}$ animals, a significant proportion of CD206+ macrophages ( 90\%) was also positive for Arg-1 (Fig. 2D). Arg-1+/CD206+ macrophages did not appreciably express iNOS as approximately $88 \%$ of these macrophages were iNOS- (Fig. 2D). In contrast, IL-6+/CD86+ macrophages were also iNOS+ $(p<0.0001$; Fig. 2E \& F). The study of the impact of IA rhPRG4 treatment on CD86+ pro-inflammatory macrophages was conducted according to the scheme presented in Supplementary Figure 1C. At 24h, rhPRG4 treatment reduced IL- $6+$ and iNOS+ CD86+ macrophages in 2-month-old Prg4 ${ }^{G T / G T}$ joints ( $p<0.05$ for both comparisons; Fig. $2 \mathrm{G} \& \mathrm{H}$ ). In contrast, PBS did not alter IL-6 or iNOS expression in CD86+ macrophages $(p>0.05$; Fig. 2G \& H).

Prg4 recombination limited the accumulation of macrophages in Prg4 $4^{G T / G T}$ joints, populated the synovium with CD86-/CD206+ (M2) macrophages, and reduced pro-inflammatory macrophages recruitment in response to TLR2 stimulation.

The Prg4 recombination studies were conducted according to the scheme presented in Supplementary Figure 1B. Gating for CD86+ and/or CD206+ macrophages was conducted as shown in Supplementary Figure 2 and Fig. 1B. Prg4 recombination reduced total macrophages in synovial tissues of 2- and 6-month-old animals $(p<$ 0.0001 for both comparisons; Fig. 3A). In addition, Prg4 recombination reduced the percentages of $\mathrm{CD} 86+1$ CD206- and CD86+/CD206+ macrophages compared to age-matched non-recombined animals $(p<0.05$ for both comparisons; Fig. 3B). Prg4 recombination also increased the percentage of CD86-/CD206+ macrophages compared to non-recombined animals $(p<0.001$; Fig. $3 \mathrm{~B})$. The modulation of CD86+ and/or CD206+ macrophages by Prg4 re-expression resulted in a lower M1/M2 ratio in synovia of 6-month-old $\operatorname{Prg} 4^{\text {GTR/GTR }}$ animals compared to age-matched Prg $4^{G T / G T}$ animals ( $p<0.01$; Fig. $3 C$ ).

The gating strategy to identify infiltrated proinflammatory macrophages, generated from circulating monocytes (characterized by positive MHC class II and Ly-6C expressions) is shown in Fig. 3D. This macrophage population had an M1 pro-inflammatory phenotype demonstrated by greater CD86 expression compared to the existing macrophage pool in $\mathrm{Prg} 4^{G T / G T}$ joints (Fig. 3E). TLR2 stimulation induced the recruitment of higher numbers of pro-inflammatory macrophages in Prg4 $4^{G T / G T}$ joints compared to Prg4 $4^{G T R / G T R}$ joints $(p<0.001$; Fig. 3F). Furthermore, TLR2 stimulation resulted in a shift towards increased CD86+/CD206- 


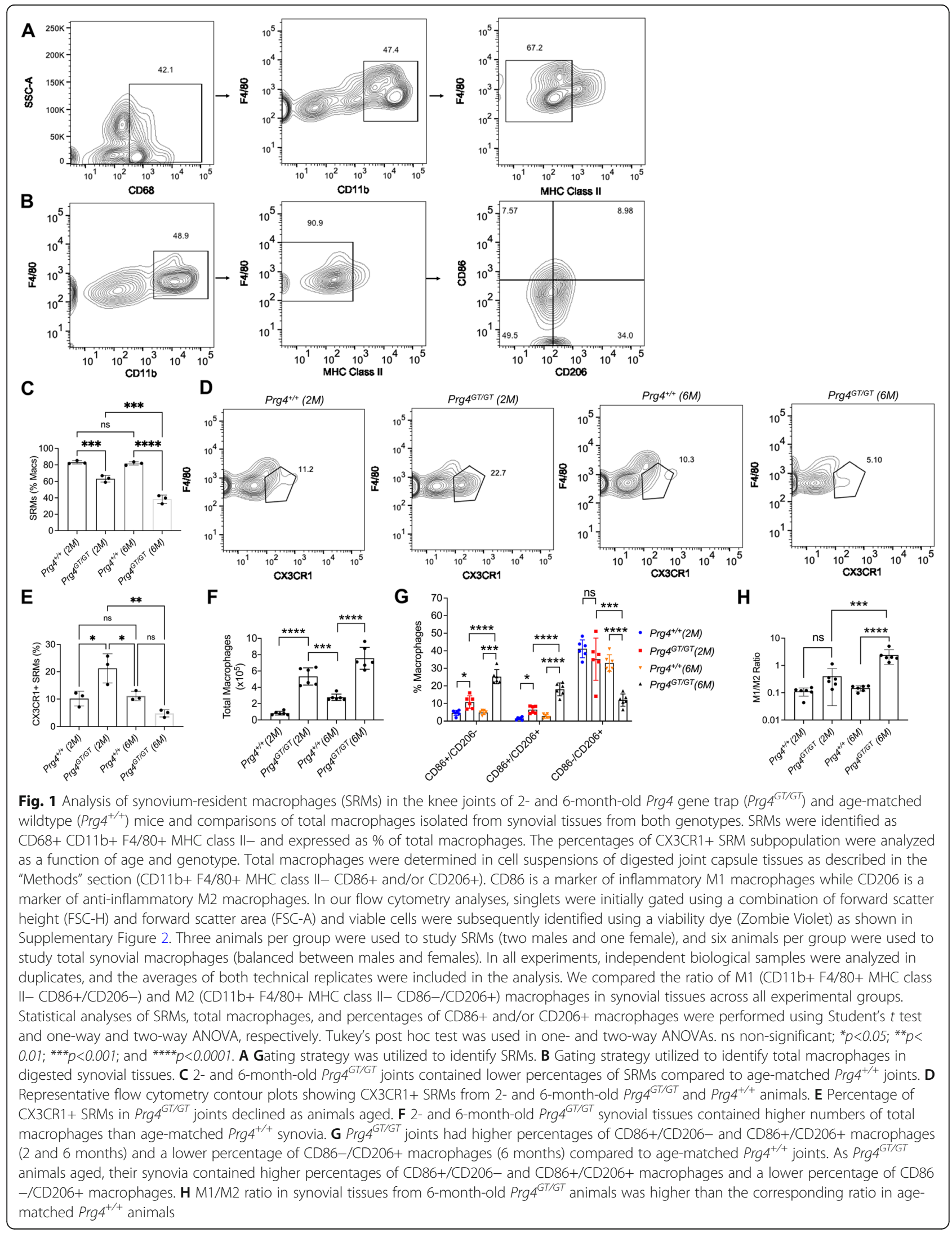




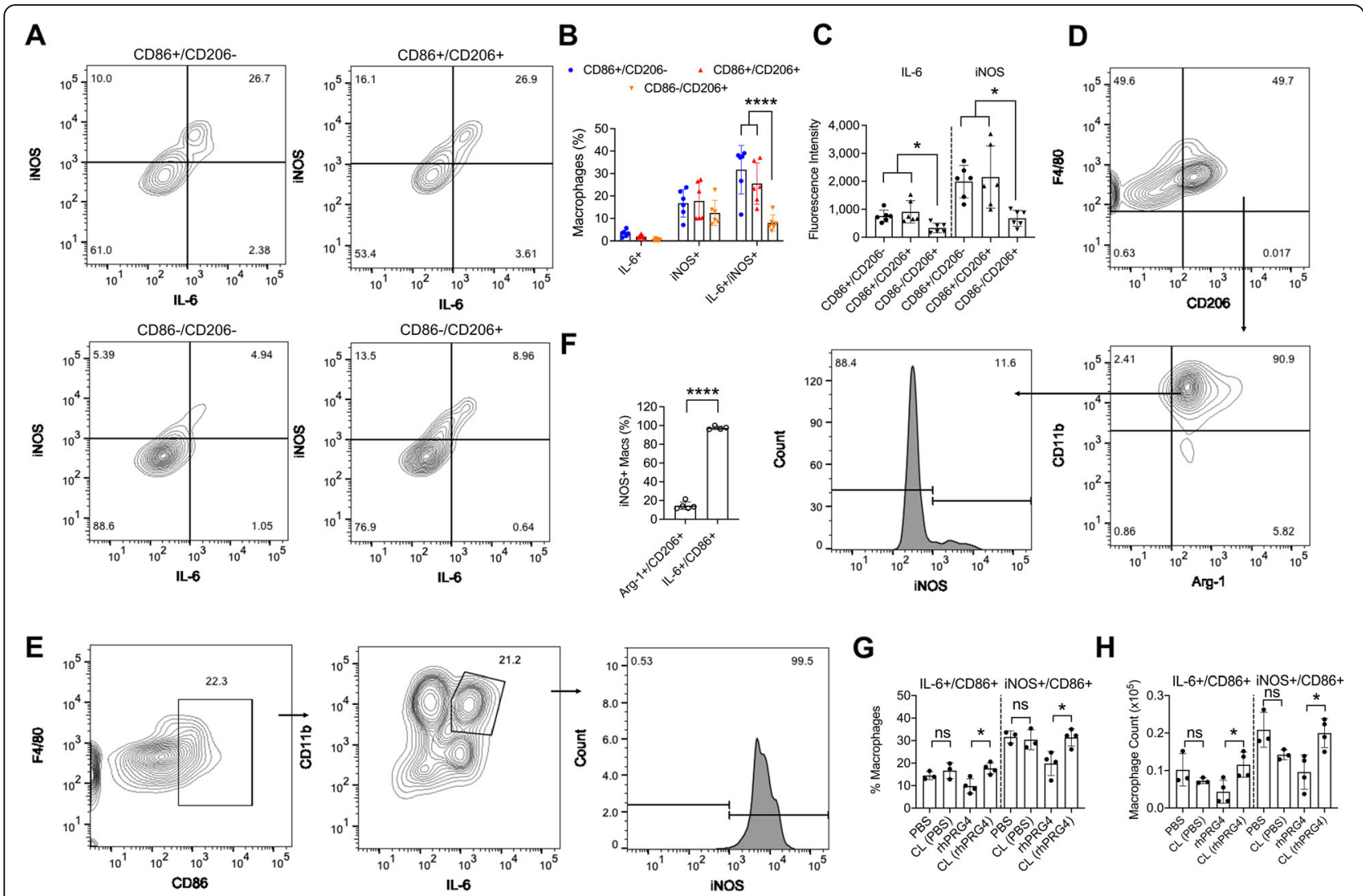

Fig. 2 Characterization of the expression of inflammatory markers, interleukin-6 (IL-6), and inducible nitric oxide synthase (iNOS) in gated synovial macrophages that express CD86 and/or CD206 surface markers from 2-month-old Prg4 gene-trap (Prg4 ${ }^{G T / G T}$ ) animals and impact of intra-articular (IA) recombinant human proteoglycan-4 (rhPRG4) treatment on IL-6 and iNOS expression in CD86+ macrophages. Macrophages were identified as CD11 b+ F4/80+ MHC class II-CD86+ and/or CD206+ and the expression levels of IL-6 and iNOS (both are markers of inflammatory M1 macrophages) and arginase-1 (Arg-1) (a marker of anti-inflammatory M2 macrophages) were evaluated using intracellular staining with PE-anti-IL6, Alexa Fluor 488-anti-iNOS, and PE-anti-Arg-1 antibodies. Positivity thresholds were set using fluorescence minus one (FMO) panel controls.

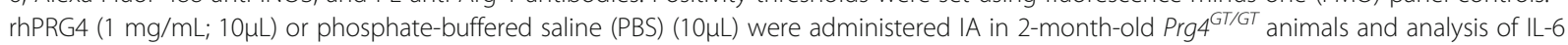
and iNOS positivity in CD11 b+ F4/80+ MHC class II-CD86+ macrophages was performed at $24 \mathrm{~h}$ following IA treatments. Macrophage cell counts were determined using precision counting beads and expressed as numbers per joint. Statistical analyses of IL-6 and iNOS staining intensities in macrophages and percentages of IL-6+ and/or iNOS+ macrophages were performed using one-way and two-way ANOVA, respectively. Tukey's post hoc test was used in one- and two-way ANOVAs. Analysis of iNOS positivity in Arg-1+/CD206+ and IL-6+/CD86+ macrophages and impact of rhPRG4 treatment was performed using unpaired and paired Student's $t$ test, respectively. Experimental groups included three ( 2 males and one female) to six animals ( 3 males and 3 females). In all experiments, independent biological samples were analyzed in duplicates, and the averages of both technical replicates were included in the analysis. ns non-significant; ${ }^{*} p<0.05$ and ${ }^{* * * *} p<0.0001$. A A representative flow cytometry contour plot of IL-6 and iNOS probing in CD86+ and/or CD206+ macrophages. Gating for CD86+ and/or CD206+ macrophages was performed according to supplementary figure 2 and fig. $1 \mathrm{~B}$ and cells in each quadrant were plotted according to their IL-6 and iNOS staining intensities, and percentages of IL-6+, iNOS+, and IL-6+/iNOS+ macrophages were determined. B Percentages of dually positive IL-6 and iNOS CD86+/CD206- and CD86+/CD206+ macrophages were higher than dually positive IL-6 and iNOS CD86-/CD206+ macrophages. C Mean IL-6 and iNOS staining intensities were higher in CD86+/CD206- and CD86+/CD206+ macrophages compared to CD86 -/CD206+ macrophages. D Gating strategy to identify Arg-1+/CD206+ macrophages in synovial tissues from 2-month-old Prg $4^{G T / G T}$ joints. Singlets and viable cells were identified as shown in Supplementary Figure 2. Viable cells were gated according to their CD206 and F4/80 expression status. Dually positive cells were then gated for Arg-1 and CD11b to identify Arg-1+/CD206+ macrophages. The percentage of the iNOS+ subset was subsequently determined. E Gating strategy to identify IL-6+/CD86+ macrophages in synovial tissues from 2-month-old $\operatorname{Prg} 4^{G T / G T}$ joints. Singlets and viable cells were identified as shown in Supplementary Figure 2. Viable cells were gated according to their CD86 and F4/80 expression status. Dually positive cells were then gated for IL-6 and CD11 b to identify IL-6+/CD86+ macrophages. The percentage of the iNOS+ subset was subsequently determined. $\mathbf{F}$ The percentage of iNOS+/IL-6+/CD86+ macrophages was higher than the corresponding percentage in iNOS+/Arg-1+/CD206+ macrophages. G rhPRG4 treatment reduced the percentages of IL-6+/CD86+ and iNOS+/CD86+ macrophages compared to contralateral $(\mathrm{CL})$ joints. No reductions were observed with PBS treatment. $\mathbf{H}$ rhPRG4 treatment reduced the numbers of IL-6+/CD86+ and iNOS+/CD86+ macrophages compared to contralateral (CL) joints. No reductions were observed with PBS treatment 


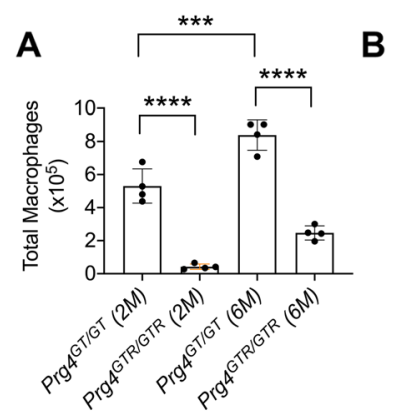

B $\cdot \operatorname{Prg} 4^{G T / G T}(6 M) \quad \sim \operatorname{Prg} 4^{G T R / G T R}(6 M) \quad$ C

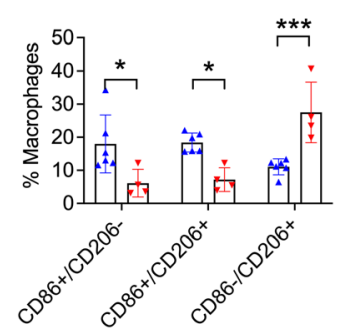

C
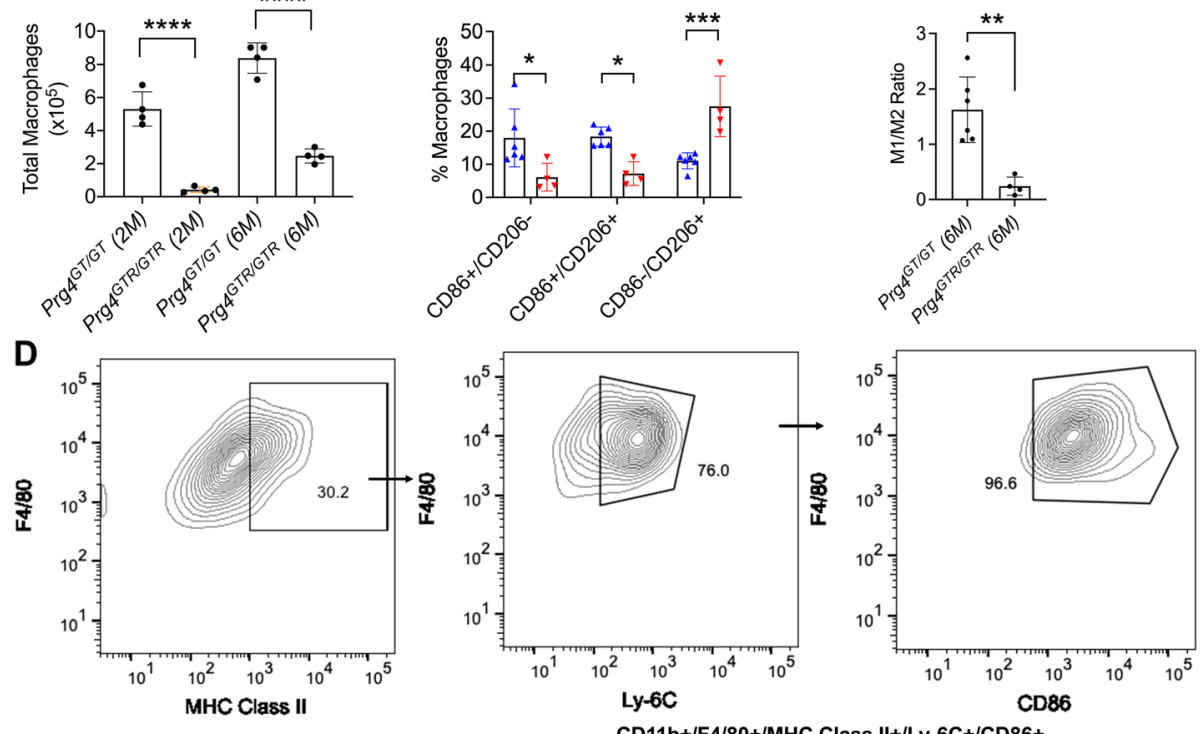

E

$F$

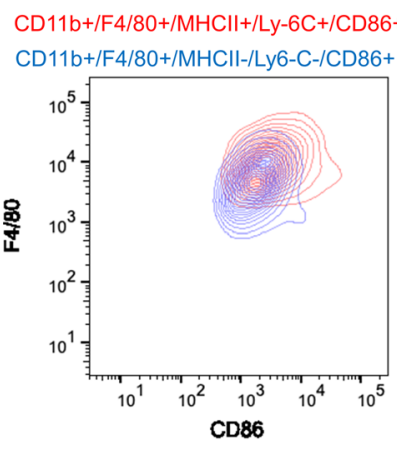

CD11b+/F4/80+/MHC Class II+/Ly-6C+/CD86+

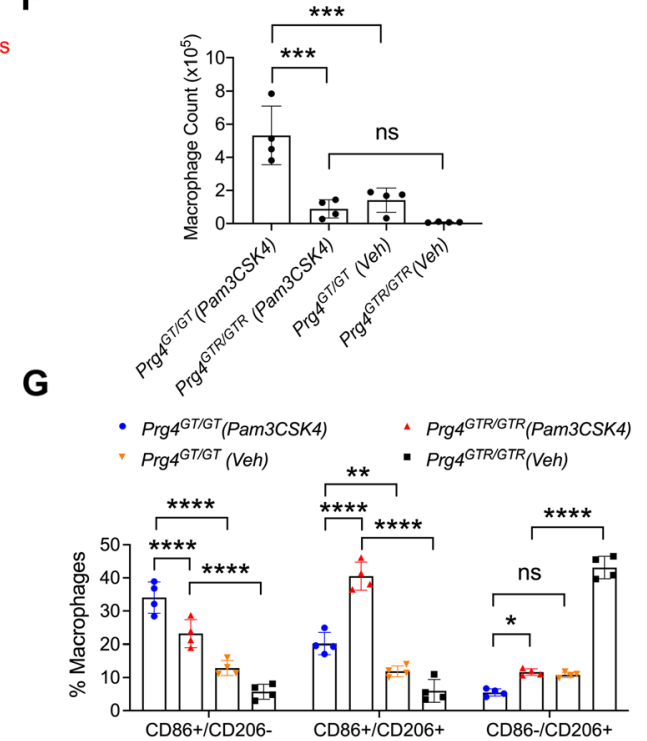

Fig. 3 (See legend on next page.) 
(See figure on previous page.)

Fig. 3 Impact of endogenous Prg4 recombination on macrophage accumulation and polarization in synovial tissues isolated from the knee joints of Prg4 gene-trap (Prg4 ${ }^{G T / G T}$ ) animals and the role of PRG4 in regulating inflammatory macrophage recruitment to the joint in response to Pam3CSK4, a Toll-like receptor 2 (TLR2) agonist. Prg4 gene recombination (Prg $4^{\text {GTR/GTR }}$ ) was performed in 3-week-old mice by intraperitoneal administration of tamoxifen $(0.1 \mathrm{mg} / \mathrm{g})$ in corn oil vehicle daily for 10 days, using vehicle-only administered age-matched Prg $4^{\text {GT/GT }}$ as controls.

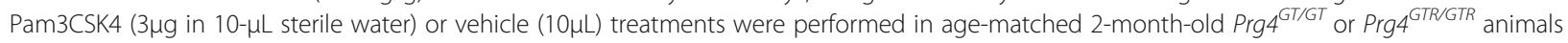
( $n=4$ per group; 2 males and 2 females), and macrophage polarization and newly infiltrated inflammatory macrophage accumulation were determined in synovial tissues at $72 \mathrm{~h}$ post-injections. Macrophages in 2- and 6-month-old Prg $4{ }^{\text {GTR/GTR }}$ animals were compared to age-matched Prg4 ${ }^{G T / G T}$ animals ( $n=4-6$ per group). Macrophages in synovial tissues were identified as CD11b+ F4/80+ MHC class $\|-$ CD86+ and/or CD206+ (using gating strategies shown in Supplementary Figure 2 and in Fig. 1B). Newly infiltrated inflammatory macrophages were identified as CD1 $1 \mathrm{~b}+$ F4/80+ MHC class II+ Ly-6C+CD86+. Macrophage cell counts were determined using precision counting beads and expressed as numbers per joint. We compared the ratio of M1 (CD11b+ F4/80+/MHC class II- CD86+/CD206-) and M2 (CD11b+ F4/80+ MHC class II- CD86-/CD206+) macrophages in synovial tissues from 6-month-old $\operatorname{Prg} 4^{G T / G T}$ and $\operatorname{Prg} 4{ }^{G T R / G T R}$ animals. In all experiments, independent biological samples were analyzed in duplicates, and the averages of both technical replicates were included in the analysis. Statistical analyses of total macrophages and percentages of CD86+ and/or CD206+ macrophages were performed using one-way and two-way ANOVA, respectively. Tukey's post hoc test was used in one- and two-way ANOVAs. ns non-significant; ${ }^{*} p<0.05$; ${ }^{*} p<0.01$; ${ }^{* *} p<0.001$; and ${ }^{* * * *} p<0.0001$. A Prg 4 gene recombination at 3 weeks reduced macrophage accumulation in 2- and 6-month-old joint tissues. B Prg $4^{G T R / G T R}$ joints at 6 months contained lower percentages of CD86+/CD206- and CD86+/CD206+ macrophages and a higher percentage of CD86-/CD206+ macrophages compared to age-matched Prg4 ${ }^{G T / G T}$ joints. C M1/M2 ratio was reduced as a consequence of Prg4 recombination. D Gating strategy to identify newly infiltrated inflammatory macrophages. Singlets and viable cells were identified as shown in Supplementary Figure 2. Cells were then gated for CD11b and F4/80 and identified MHC class II+ Ly-6C+ CD86+ population as shown in the representative flow cytometry contour plot. E Representative flow cytometry contour plot depicting stronger CD86 epitope staining in newly infiltrated inflammatory macrophages compared to the existing macrophage populations in synovial tissues of Pam3CSK4-administered Prg $4^{\text {GT/GT }}$ joints. F Pam3CSK4 administration resulted in greater inflammatory macrophage recruitment in 2-month-old Prg $4^{\text {GT/GT }}$ joints compared to Prg $4^{\text {GTR/GTR }}$ joints. G Pam3CSK4 administration resulted in a shift towards an inflammatory CD86+ macrophage, particularly in 2-month-old Prg $4^{G T / G T}$ joints

macrophages and reduced CD86-/CD206+ macrophages in $\operatorname{Prg} 4^{G T / G T}$ joints compared to $\operatorname{Prg} 4^{\text {GTR/GTR }}$ joints $(p<$ $0.0001 ; p<0.05$; Fig. 3G).

TLR2-stimulated M2a BMDMs from Prg4 ${ }^{G T / G T}$ animals exhibited elevated expressions of inflammatory cytokines and rhPRG4 treatment reduced the secretion of cytokines and chemokines by these BMDMs.

M2a BMDMs from Prg4 ${ }^{G T / G T}$ animals had higher expression levels of IL-1 $\beta$ and IL-6 and a lower expression level of IL-10 following TLR2 stimulation ( $p<0.05$ for all comparisons; Fig. 4A). While TLR2 stimulation induced iNOS expression in BMDMs from Prg $4^{G T / G T}$ and wildtype animals, there was no difference in iNOS expression levels between the two genotypes ( $p>0.05$; Fig. $4 \mathrm{~A})$. rhPRG4 treatment reduced TLR2 agonist-induced expression of iNOS, IL-1 $\beta$, and IL-6 $(p<0.05$ for all comparisons) with no effect on IL-10 expression in Prg $4^{G T / G T}$ M2a BMDMs (Fig. 4B). Representative proteome profiler blots of Pam3CSK4 \pm rhPRG4-treated BMDMs showing reducing signal intensities of select cytokines and chemokines are depicted in Fig. 4C. The proteome profiler cytokine array map is shown in Supplementary Figure 3. rhPRG4 treatment reduced secreted levels of inflammatory cytokine: IL-6 $(p<0.0001)$ and TNF- $\alpha(p<0.0001)$ and chemokines: CXCL1/GRO- $\alpha$ $(p<0.001), \quad$ CXCL2/MIP-2 $\quad(p<0.0001), \quad$ CXCL10 $\quad(p<$ $0.001), \quad$ CCL2/MCP-1 $\quad(p<0.001), \quad$ CCL3/MIP- $\alpha \quad(p<$ $0.0001)$, CCL4/MIP- $\beta(p<0.0001)$, and CCL5/RANTES $(p<0.0001)$ (Fig. 4D).

\section{Depletion of macrophages reversed synovial fibrosis in $\mathrm{Prg} 4{ }^{\mathrm{GT} / \mathrm{GT}}$ knee joints}

Macrophage depletion studies were conducted according to the scheme presented in Supplementary Figure 1D. Gating of macrophage populations of interest was performed as described in Supplementary Figure 2 and Fig. 1B. In our intermediate endpoint study (conducted on day 15 following treatment initiation), we observed that clodronate liposome treatment altered the distribution of CD86+ and/or CD206+ macrophages in synovial tissues towards a reduction in CD86+ and/or CD206+ fractions, and a reduction in the number of events classified as CD11b+ F4/80+ MHC class II- cells (Fig. 5A), consistent with a depleting effect on synovial macrophages. As a consequence of clodronate liposome treatment, the percentages of CD86+ and CD86-/CD206+ macrophages were reduced compared to PBS liposome treatment $(p<0.0001$ for both comparisons; Fig. 5B). As shown above, CD86+ macrophages populated the synovium as animals aged, and as such, they may be causally implicated in the progressive development of synovial hyperplasia and fibrosis. Therefore, we studied the impact of clodronate on CD86+ macrophage numbers in $\operatorname{Prg} 4^{G T / G T}$ synovia and detected a reduction that approximated $62 \%$ in magnitude ( $p<0.01$; Fig. $5 \mathrm{C})$. In our histology endpoint studies (conducted on day 30 following treatment initiation), we observed more significant macrophage depletion demonstrated by fewer immune cells in H\&E-stained sections and reduced CD11b 


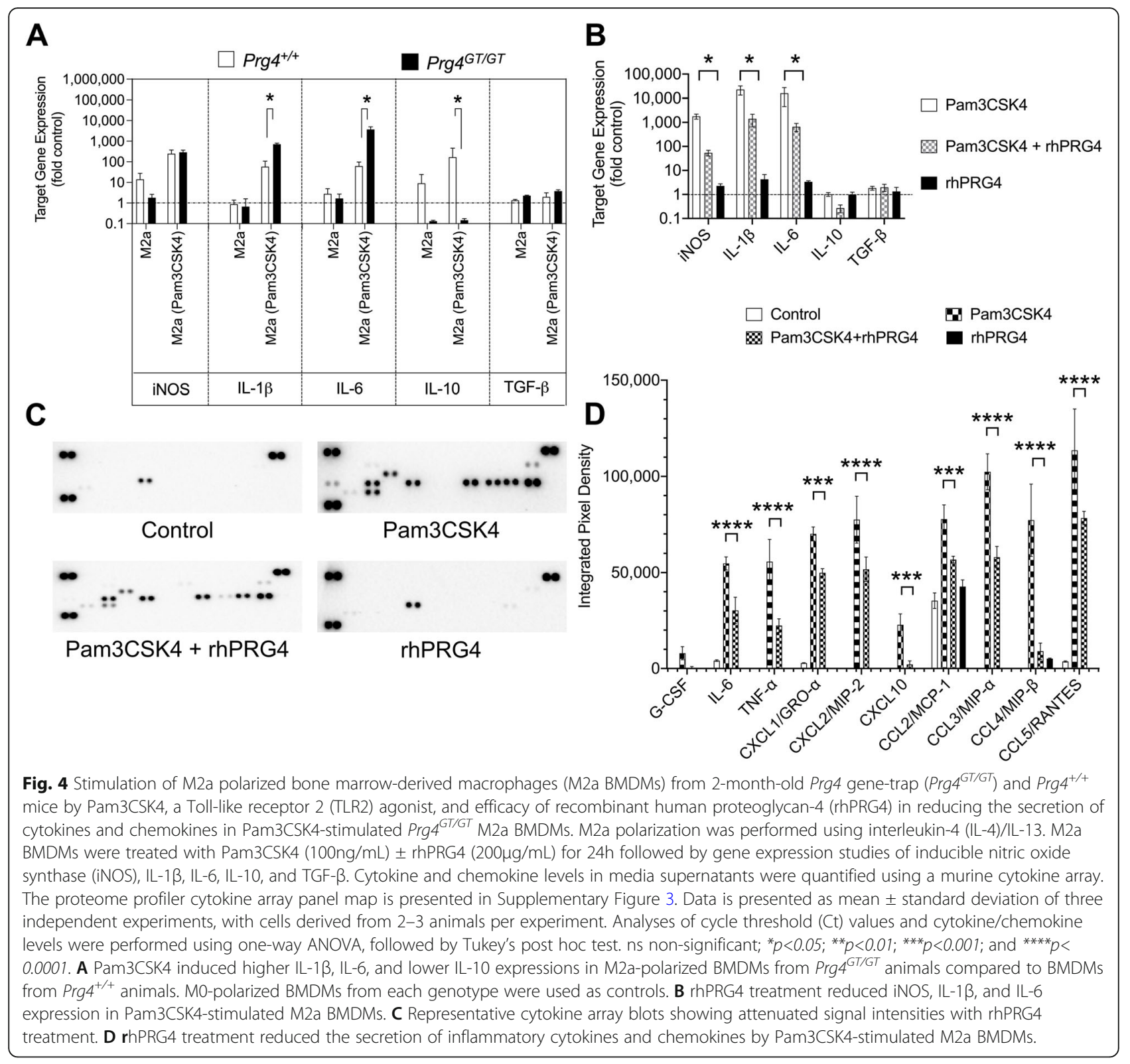

staining in synovial tissues of clodronate-treated Prg $4^{G T / G T}$ joints $(p<0.0001$; Fig. 5D \& F). Synovial membrane thickness was also reduced $(p<0.0001)$ with clodronate liposomes (Fig. 5E). In addition, no appreciable $\alpha$-SMA, PLOD2, or COL-I staining was detected in synovial tissues of clodronate liposome-treated $\operatorname{Prg} 4^{G T / G T}$ joints, while these markers were detected in synovial tissues of PBS liposomes-treated Prg4 $4^{G T / G T}$ joints $(p<0.001$; $p<0.0001 ; p<0.01$; Fig. 5D \& F).

\section{Discussion}

In this work, we investigated the temporal changes in tissue-resident and total macrophages in synovial tissues from joints lacking Prg4 expression and discovered that the tissue-resident fraction of macrophages decreased with age along with a simultaneous increase in the total number of macrophages, indicating that macrophages of a non-resident origin have progressively invaded and populated Prg4 null synovial tissues. In the Prg4 null synovium, the totality of macrophages exhibited an agedependent shift towards the pro-inflammatory M1 phenotype and away from the anti-inflammatory M2 phenotype, and as such, a dramatically high $\mathrm{M} 1 / \mathrm{M} 2$ ratio was observed in 6-month-old Prg4 null synovia that coincided with significant synovial pathology. Early in the development of synovial pathology, synoviumresident macrophages appeared to be enriched in the anti-inflammatory CX3CR1+ SRM subtype, potentially 


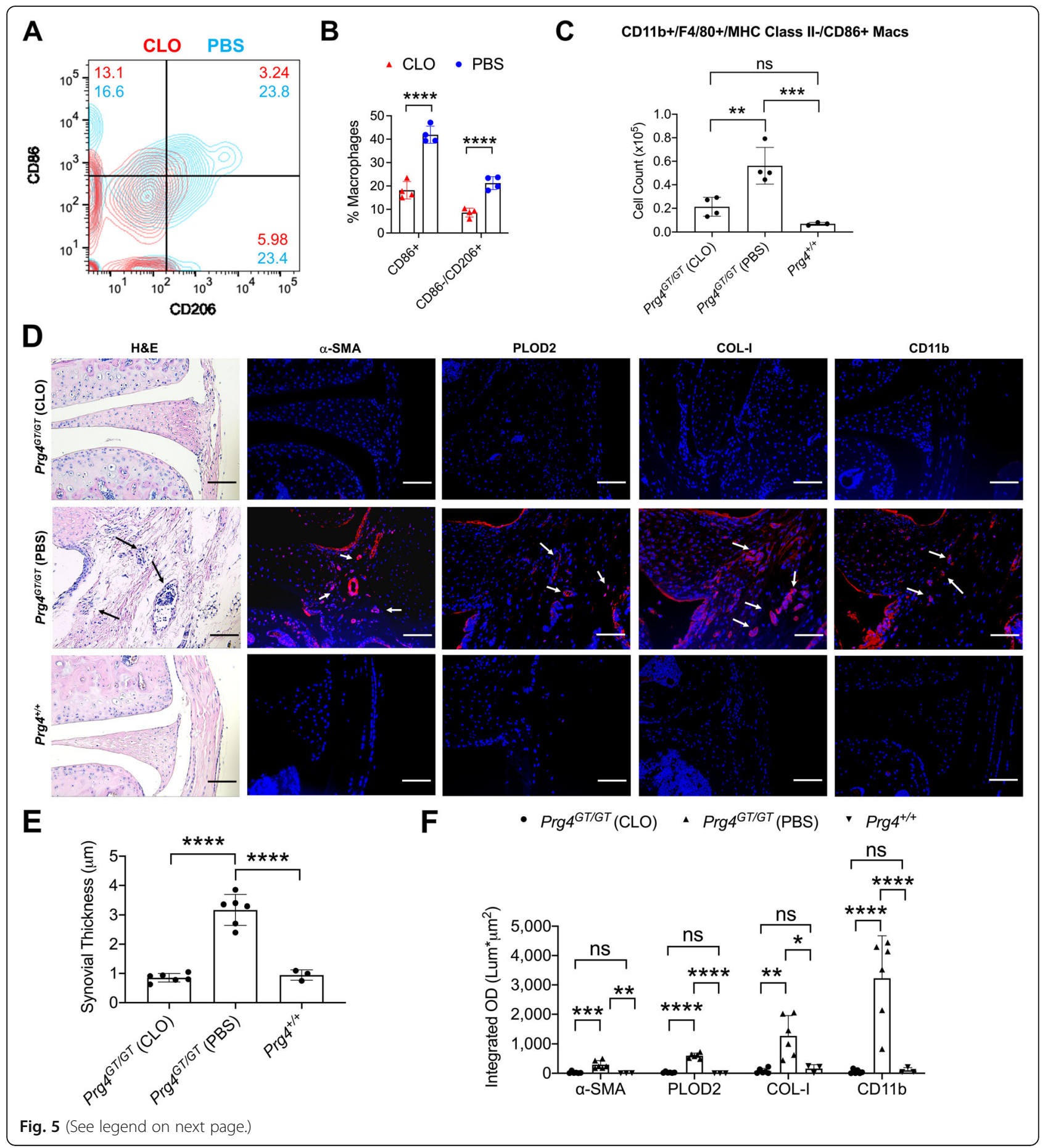




\begin{abstract}
(See figure on previous page.)
Fig. 5 Impact of macrophage depletion on synovial pathology in 4-month-old Prg4 gene-trap (Prg $\left.4^{G T / G T}\right)$ animals. The knee joints of Prg $4^{G T / G T}$

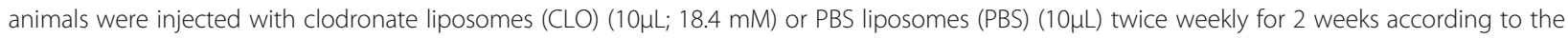
scheme presented in Supplementary Figure 1D. The percentages of CD11b+ F4/80+ MHC class II- CD86+ or CD86-/CD206+ macrophages were determined in cell suspensions from digested joint capsule tissues on day 15 following treatment initiation, and the numbers of CD86+ macrophages were determined using precision counting beads. Cell gating was performed as described in Supplementary Figure 2 and Fig. 1B. In a separate study, synovial pathology measurements were performed on day 30 following treatment initiation and included synovial thickness and staining intensities of fibrosis markers: alpha-smooth muscle actin (a-SMA), PLOD2, and collagen type I (COL-I) as well as the monocyte/ macrophage marker CD11b ( $n=6$ per group). Age-matched Prg4 wildtype animals (Prg ${ }^{+/+}, n=3$ ) were used as controls. Analyses of macrophage cell count, synovial thickness, a-SMA, PLOD2, COL-I, and CD11b staining intensities were performed using one-way ANOVA followed by Tukey's post hoc test. Analysis of CD86+ or CD86-/CD206+ macrophages was performed using Student's $t$ test. Scale $=10 \mu m$. ns non-significant; ${ }^{*} p<$ 0.05 ; ${ }^{* *} p<0.01$; ${ }^{* * *} p<0.001$; and ${ }^{* * * *} p<0.0001$. A Representative contour plot of CD86 and CD206 staining in macrophages of synovial tissues from CLO- or PBS-treated joints at 15 days following treatment initiation. The most prominent change with CLO treatment was a reduction in the percentage of CD86+/CD206+ macrophages. B Percentages of CD86+ and CD86-/CD206+ macrophages were reduced with CLO treatment. C Inflammatory macrophages in Prg $4^{G T / G T}$ joints were depleted with CLO treatment. D Representative histological sections (H\&E, a-SMA, PLOD2, COL-I, and CD11b) depict thickening of the synovial membrane, immune cell infiltration, positive a-SMA, PLOD2, COL-I, and CD11b staining (all indicated by arrows) in PBS-treated joints. Sections were counterstained with DAPI. Macrophage depletion reduced synovial thickness in Prg4 ${ }^{\text {GT/GT }}$ joints. E Macrophage depletion reduced a-SMA, PLOD2, COL-I, and CD11b staining in Prg ${ }^{G T / G T}$ joints
\end{abstract}

to compensate for the emergence of M1 macrophages in $\operatorname{Prg} 4^{G T / G T}$ synovia. However, with age and as synovitis persisted, the CX3CR1+ subset diminished, signaling a compromised endogenous anti-inflammatory role for synovial macrophages. A macrophage population that was positive for both CD86 and CD206 was identified in $\operatorname{Prg} 4^{G T / G T}$ synovial tissues, and this population increased with age further supporting that in vivo, macrophages can exist with a high degree of plasticity, where both M1 and M2 markers are expressed by the same cell. For this specific macrophage subtype, it was unclear whether it is predominantly pro- or anti-inflammatory. To investigate this, we probed for other functional M1 markers, e.g., IL-6 and iNOS and concluded that CD86+/CD206+ macrophages have a pro-inflammatory phenotype, indicated by enhanced IL- 6 and iNOS expressions, that is comparable to CD86+ only macrophages. It is therefore reasonable to assume that this macrophage population plays an effector role, together with CD86+/CD206macrophages, in mediating chronic synovitis in Prg4 null joints. CD86+/CD206+ macrophages might have originated from CD86-/CD206+ counterparts in the synovium. This notion is supported by our observation that in TLR2 agonist-treated Prg recombined joints, the reduction in CD86-/CD206+ macrophages was accompanied by a comparable increase in CD86+/CD206+ macrophages, suggesting that M2 macrophages expressed CD86 in response to TLR2 stimulation. This phenomenon has been previously reported in human macrophages where M2 macrophages displayed M1 markers in response to TLR2 stimulation [41], and these macrophages had a pro-inflammatory phenotype as well [41]. The involvement of TLR2 signaling in the generation of CD86+/CD206+ macrophages is also supported by observations that TLR2 activation is a requirement for CD86 upregulation in murine macrophages and subsequent Th1 immune response in vitro and in vivo [42].

Prg4 recombination re-established synovial macrophage homeostasis as it enriched the synovium with the anti-inflammatory M2 macrophages and prevented M1 macrophage accumulation in the synovium and as such modified the M1/M2 ratio to be in line with the corresponding ratio in age-matched wild type tissues. Exogenous PRG4 administration in the joints lacking Prg4 expression immediately attenuated the pro-inflammatory M1 macrophage phenotype as it reduced iNOS and IL-6 expressions in CD86+ macrophages, arguing for a direct effect of PRG4 on macrophages. To further explore this assumption, we supplemented our in vivo studies with an in vitro investigation into whether PRG4 can directly modulate macrophage responsiveness to inflammatory stimuli. We detected an impaired anti-inflammatory activity of M2 macrophages from Prg4 null animals evidenced by a reduced IL-10 and increased IL-1 $\beta$ and IL-6 expressions. Furthermore, rhPRG4 reduced the expression and secretion of inflammatory cytokines by TLR2-stimulated M2a BMDMs. Hence, our in vitro experiments support that PRG4 regulates macrophage activation and that its in vivo homeostatic role in the joint might be facilitated by a direct effect on synovial macrophages. However, the biological effect of PRG4 on synovial fibroblasts in the context of attenuating synovial pathology characteristics of Prg4 null mice should also be considered. PRG4 exerts direct anti-inflammatory, anti-proliferative, and anti-fibrotic effects on synovial fibroblasts [22, 23], and as such, regulation of synovial fibroblasts is a potential mechanistic component of PRG4's homeostatic role in the synovium. PRG4 also has a lubricating mechanical role in the joint, and the absence of such a role might contribute to the dysregulated 
synovial macrophage homeostasis observed in Prg4 null mice. Nonetheless, PRG4's effect on macrophages in vivo might be biologically significant, specifically in the context of arresting inflammation and related synovial hyperplasia and fibrosis. Our work with macrophage depletion lends support to the important role of macrophages in regulating synovial pathology in Prg4 null mice. Independent of PRG4, synovial macrophage depletion reduced synovial hyperplasia and fibrosis consistent with the hypothesis that an aberrant pattern of macrophage polarization in the absence of PRG4 is what mediates chronic synovitis in Prg4 null joints.

Our understanding of the contribution of macrophages to synovial fibrosis is severely limited. In other tissues, distinct macrophage populations appear to coordinate the initiation, maintenance, and resolution of inflammation and its associated tissue repair [43]. Tissue-resident macrophages maintain tissue immune homeostasis [3, 4] and successful resolution of inflammation requires coordination between the switching of pro-inflammatory macrophages to an anti-inflammatory phenotype and the activation of the tissue-resident macrophage pool to aid in tissue repair [4, 44]. In our $\operatorname{Prg} 4^{G T / G T}$ model, inflammation was not adequately resolved, which is rationalized by a diminished antiinflammatory tissue-resident macrophage population and a lack of differentiation of M1 macrophages to M2 macrophages. The inadequate resolution of chronic inflammation in Prg4 null joints contributed to dysregulated tissue repair and hence the development of synovial fibrosis that progressed with age [23]. Since a significant fraction of macrophages in the synovium was pro-inflammatory, we aimed to evaluate whether depletion of those pro-inflammatory macrophages alters the trajectory of synovial hyperplasia and fibrosis. We utilized liposomal clodronate to deplete macrophages in the synovium since liposomes facilitate the internalization of the hydrophilic clodronate in phagocytic macrophages where clodronate causes their apoptosis [45]. IA clodronate liposomes have successfully been used in depleting synovial macrophages where macrophage depletion can be seen 7 days following IA administration [45, 46]. Clodronate liposomes were used to deplete macrophages in a murine surgically induced experimental OA model $[46,47]$. In one report, a single dose of clodronate liposomes depleted synovial-lining macrophages and reduced MMP-3-mediated cartilage degeneration [46], while in another study, a 3-dose regimen over 9 weeks depleted macrophages across the entire thickness of the synovium and reduced synovitis scores and synovial thickness [47]. In our model, we utilized a 4-dose regimen over 2 weeks and assessed early changes to the macrophage pool in $\operatorname{Prg} 4^{G T / G T}$ synovia. Clodronate treatment appeared to reduce the fraction of pro- inflammatory and anti-inflammatory macrophages in the synovium. At the time of our histological analyses, macrophages were depleted from the entire thickness of the synovial tissues along with suppressed fibrotic markers' expression and reduced synovial hyperplasia. Since clodronate treatment resulted in pan macrophage depletion, it is hard to attribute its resolving effect on fibrosis to the depletion of a specific macrophage population. Nonetheless, it is argued that since the predominant macrophage phenotype in the synovium at the time of clodronate administration is pro-inflammatory, clodronate may have exerted its effect via suppressing synovial inflammation due to a significant depleting effect on pro-inflammatory macrophages in the synovium. Our findings collectively highlight that synovial hyperplasia and fibrosis in joints lacking Prg4 expression are potentially propagated by an enhanced shift towards proinflammatory macrophages and that re-establishing macrophage phenotypic homeostasis either via Prg4 recombination [23] or via depleting synovial macrophages is efficacious in attenuating synovial pathology.

Circulating monocyte recruitment and differentiation into pro-inflammatory macrophages in the synovium adds to the existing M1 macrophage pool and exacerbates synovitis [12]. In our study, the lack of Prg4 expression in the synovium enhanced monocyte recruitment and differentiation into pro-inflammatory macrophages, and Prg4 recombination limited the accumulation of infiltrated macrophages into the joint. The magnitude by which proinflammatory macrophages populate the synovium, linked to Prg4 expression status, may explain how PRG4 regulates the recruitment of monocyte-derived proinflammatory macrophages. A larger M1 inflammatory pool in the synovium, as seen in Prg4 null mice, may generate higher quantities of chemokines in the joint in response to TLR2 stimulation and thus increase monocyte recruitment. While we have not specifically measured the secretion of chemokines in Prg null and competent joints, we aimed to study whether PRG4 modulates chemokine secretion by BMDMs in vitro. We observed that BMDMs secrete chemokines of the CXC and CC families [48] to variable degrees. CXC chemokines attract neutrophils, lymphocytes, and monocytes to the synovium [48], while CC chemokines attract monocytes, $\mathrm{T}$ cells, and natural killer cells [48]. rhPRG4 reduced, by a biologically significant magnitude, the secretion of multiple chemokines with the greatest effect shown for CCL4. Overall, the magnitude of chemokine reduction with rhPRG4 treatment appeared to be greater with CCL2, CCL3, CCL4, and CCL5 compared to CXCL1 and CXCL2. The ability of rhPRG4 to attenuate chemokine secretion by activated macrophages may provide a plausible explanation of why we observed a reduction in monocyte infiltration following Prg4 recombination. 
It is clear that the dichotomous nature of M1-M2 macrophage polarization is insufficient to adequately describe the multifaceted and complex functions of macrophages in the synovium [49]. However, it remains useful to investigate changes in the phenotypic balance of macrophages according to the broad M1-M2 classification, especially if they can be correlated to structural and pathological changes at the tissue level. We have selected two markers for M1 and M2 macrophages that have been used to study macrophage phenotypic balance in synovial tissues of OA patients and pre-clinical surgically induced OA models [49]. We have also used complementary markers, e.g., IL-6 and iNOS to confirm the pro-inflammatory phenotype of macrophage populations of interest. Arg-1 was also used as a confirmatory marker of the M2 phenotype of CD206+ macrophages in 2-month-old animals where the majority of CD206+ macrophages lacked CD86 expression. The changes in macrophage phenotypic balance that we observed in our gene-trap animals with age trended in a similar direction to what has been reported in the murine DMM model where M1 macrophages (F4/80+ CD86+ CD63-) were increased in the synovium at 6 weeks following DMM surgery [50], and M2 macrophages (F4/80+ CD11c+ CD206+) were reduced at 8 weeks following DMM surgery, along with an increase in iNOS+ macrophages [51]. These DMM synovial macrophage studies in conjunction with our current findings provide a strong motivation to investigate the role of PRG4 in regulating synovial macrophage polarization in $\mathrm{OA}$ as the basis for its disease-modifying activity $[27,28]$. Our study has a number of limitations. One limitation is that we did not study the impact of macrophage depletion on cartilage health in $\operatorname{Prg} 4^{G T / G T}$ joints. In addition, we did not investigate the impact of a joint injury, e.g., DMM on synovial macrophage polarization in Prg4 null and competent mice. We focused our effort on analyzing macrophages in the synovium and faced technical challenges, and we did not characterize macrophages in other joint compartments, e.g., the synovial fluid. Joint fluid macrophages might have a role in the synovial pathology of Prg $4^{G T / G T}$ animals. However, macrophages in the synovium, due to their physical proximity, would reasonably exert the greatest influence on synovial hyperplasia and fibrosis. In this study, we were not able to sort out the hybrid M1/M2 (CD86+ CD206+) macrophage population in high-enough yield to perform an extensive comparative phenotypic characterization to M1 (CD86+ CD206-) and M2 (CD86- CD206+) macrophages. Finally, we did not compare the extent of IL-6 and iNOS expressions in M1 macrophages from $\operatorname{Prg} 4^{G T / G T}$ animals to those in M1 macrophages from wild-type animals following a traumatic joint insult.

\section{Conclusion}

In summary, we have shown that PRG4 plays an important role in maintaining synovial macrophage homeostasis, where a lack of Prg4 expression increases total macrophage numbers in the synovium, with a pattern of abundant pro-inflammatory macrophages and diminished antiinflammatory macrophages. As pro-inflammatory macrophages populate the synovium, structural changes, e.g., synovial hyperplasia and fibrosis, become significant and macrophage depletion reverses this synovial pathology. Given that an imbalance between pro-inflammatory and anti-inflammatory synovial macrophages has been reported in patients with OA and pre-clinical OA models, novel treatments that target macrophage polarization to re-establish homeostasis either directly or indirectly via synovial PRG4 upregulation may prove beneficial in treating chronic synovitis and thus slowing OA progression.

\section{Abbreviations}

a-SMA: Alpha smooth muscle actin; ANOVA: Analysis of variance; CD44: Cluster determinant 44; COL-1: Collagen type-1; Ct: Cycle threshold; CX3CR1: C-X3-C motif chemokine receptor 1; DAMPs: Damage-associated molecular patterns; DMEM: Dulbecco's modified Eagle's medium; DMM: Destabilization of the medial meniscus; ELISA: Enzyme-linked immunosorbent assay; GAPDH: Glyceraldehyde-3-phosphate dehydrogenase; IL-1 $\beta$ : Interleukin-1 beta; IL-1Ra: Interleukin-1 receptor antagonist; IL6: Interleukin-6; IL-10: Interleukin-10; iNOS: Inducible nitric oxide synthase; MCP-1: Monocyte chemoattractant protein-1; MHC class II: Major histocompatibility complex class II; MMPs: Matrix metalloproteinases; NFKB: Nuclear factor kappa b; OA: Osteoarthritis; PBS: Phosphate-buffered saline; PCR: Polymerase chain reaction; PLOD2: Procollagen-lysine, 2-oxogluterate 5dioxygenase 2; PRG4: Proteoglycan-4; PTOS: Posttraumatic osteoarthritis;

RA: Rheumatoid arthritis; rhPRG4: Recombinant human PRG4;

SRMs: Synovium-resident macrophages; SM: Synovial macrophages;

TLR2: Toll-like receptor 2; TLR4: Toll-like receptor 4; TGF- 31 : Transforming growth factor beta 1

\section{Supplementary Information}

The online version contains supplementary material available at https://doi. org/10.1186/s13075-021-02621-9.

Additional file 1: Supplementary Figure 1. Overview of experimental design for in vivo studies. In our experiments, we pooled joint capsular tissues from two knee joints and identified this sample as an independent biological sample. In experiments where knee joints did not receive a treatment, the right and left knee joints were pooled from the same animal to generate an independent biological replicate. *In these experiments, the " $n$ " refers to independent biological replicates generated by pooling tissues from two animals. Unless otherwise specified, each experimental group contained equal numbers of males and females.

Additional file 2: Supplementary Figure 2. Gating strategy to identify singlets and viable cells using Zombie Violet viability dye. Total cells were gated based on forward scatter area (FSC-A) and side scatter area (SSC-A). Subsequently, singlets were identified based on a combination of FSC-A and forward scatter height (FSC-H). Viable cells were identified using Zombie Violet viability dye

Additional file 3: Supplementary Figure 3. Proteome Profiler Mouse Cytokine Array Panel A Map. Ref. Spot: Reference Spot; Neg. Control: Negative Control (PBS). 


\section{Authors' contributions}

$M Q$ and $K E$ conceived the study. MQ, LZ, GJ, JT, and KE carried out the experiments and participated in the analysis of the data. TS provided the reagents and critical interpretation of the results. The authors have participated in drafting and critical evaluation of the manuscript. The authors have read and approved the final version of the manuscript.

\section{Authors' information}

Marwa Qadri, Pharm.D., Ph.D.: Assistant Professor of Pharmacology, Jazan University School of Pharmacy, Jazan, Kingdom of Saudi Arabia. Gregory D. Jay, MD, Ph.D.: Professor, Emergency Medicine and Engineering, Brown University, Providence, RI, USA.

Ling Zhang, MD: Senior Research Assistant, Rhode Island Hospital, Providence, RI, USA.

Holly Richendrfer: Research Associate, Rhode Island Hospital, Providence, RI, USA.

Tannin A. Schmidt, Ph.D.: Associate Professor of Biomedical Engineering, University of Connecticut Health Center, Farmington, CT, USA. Jennifer Totonchy, Ph.D.: Assistant Professor of Biomedical and Pharmaceutical Sciences, Chapman University, Irvine, CA, USA. Khaled A. Elsaid, Pharm.D, Ph.D.: Associate Professor of Biomedical and Pharmaceutical Sciences, Chapman University, Irvine, CA, USA.

\section{Funding}

This work is supported by R01AR067748 to KE and GJ.

\section{Availability of data and materials}

Not applicable

\section{Declarations}

\section{Ethics approval and consent to participate}

Harvest of joint capsular tissues from mice, intra-articular administration of Pam3CSK4, liposomal clodronate and PBS, and histological evaluations of mice joints and harvest of murine bone marrows were approved by the IACUC committee at Chapman University. All experiments were performed in accordance with the established guidelines and regulations.

\section{Consent for publication}

Not applicable

\section{Competing interests}

$M Q$, LZ, and JT have nothing to disclose.

GJ authored patents on rhPRG4 and holds equity in Lubris LLC, MA, USA.

ATS authored patents on rhPRG4, is a paid consultant for Lubris LLC, MA,

USA, and holds equity in Lubris LLC, MA, USA.

KE authored patents on rhPRG4.

The authors have no non-financial competing interests related to this manuscript.

\section{Author details}

'Department of Pharmacology, College of Pharmacy, Jazan University, Jazan 82826, Kingdom of Saudi Arabia. 'Department of Emergency Medicine, Rhode Island Hospital, Providence, RI, USA. ${ }^{3}$ Biomedical Engineering Department, School of Dental Medicine, University of Connecticut, Farmington, CT, USA. ${ }^{4}$ Department of Biomedical and Pharmaceutical Sciences, Chapman University School of Pharmacy, Rinker Health Sciences Campus, 9401 Jeronimo Road, Irvine, CA 92618, USA

\section{Received: 6 April 2021 Accepted: 5 September 2021}

Published online: 14 September 2021

\section{References}

1. Smith MD. The normal synovium. Open Rheumatol J. 2011;5:100-6.

2. Smith MD, Barg E, Weedon $H$, Papengellis V, Smeets $T$, Tak $P$, et al. Microarchitecture and protective mechanisms in synovial tissue from clinically and arthroscopically normal knee joints. Ann Rheum Dis. 2003;62: 303-7.

3. Davies LC, Taylor PR. Tissue-resident macrophages: then and now Immunology. 2015;144(4):541-8.
4. Davies LC, Jenkin SJ, Allen JE, Taylor PR. Tissue-resident macrophages. Nat Immunol. 2013:14(10):986-95.

5. Culemann S, Grüneboom A, Nicolas-Avila JA, Weidner D, Lammie KF, Rothe $T$, et al. Locally renewing resident synovial macrophages provide a protective barrier for the joint. Nature. 2019;572(7771):670-5.

6. Bondeson J, Blom AB, Wainwright S, Hughes C, Caterson B, van den Berg WB. The role of synovial macrophages and macrophage-produced mediators in driving inflammatory and destructive responses in osteoarthritis. Arthritis Rheum. 2010;62(3):647-57.

7. Kraus VB, McDaniel G, Huebner JL, Stabler TV, Pieper CF, Shipes SW, et al. Direct in vivo evidence of activated macrophages in human osteoarthritis. Osteoarthritis Cartilage. 2016;24(9):1613-21.

8. Kennedy A, Fearson U, Veale DJ, Godson C. Macrophages in synovial inflammation. Front Immunol. 2011;2:52

9. Wang $\mathrm{N}$, Liang $\mathrm{H}$, Zen K. Molecular mechanisms that influence the macrophage M1-M2 polarization balance. Fron Immunol. 2014;5:1-9.

10. Ley K. M1 means kill; M2 means heal. J Immunol. 2017;199:2191-3.

11. Donlin LT, Jayatilleke A, Giannopoulou EG, Kalliolias GD, Ivashkiv LB. Modulation of TNF-induced macrophage polarization by synovial fibroblasts. J Immunol. 2014;193(5):23773-2383.

12. Zhang H, Lin C, Zeng C, Wang Z, Wang H, Lu J, et al. Synovial macrophage M1 polarisation exacerbates experimental osteoarthritis partially through Rspondin-2. Ann Rheum Dis. 2018;77(10):1524-34.

13. Orecchioni M, Ghosheh Y, Pramond AB, Ley K. Macrophage polarization: different gene signatures in M1 (LPS+) vs. classically and M2 (LPS-) vs. alternatively activated macrophages. Front Immunol. 2019;10:1084.

14. Huang QQ, Birkett R, Doyle RE, Haines GK, Perlman H, Shi B, et al. Association of increased $\mathrm{F} 4 / 80^{\text {high }}$ macrophages with suppression of serumtransfer arthritis in mice with reduced FLIP in myeloid cells. Arthritis Rheumatol. 2017:69(9):1762-71.

15. Ambarus CA, Noordenbos T, de Hair MJ, Tak PP, Baeten DL. Intimal lining layer macrophages but not synovial sublining macrophages display an IL-10 polarized-like phenotype in chronic synovitis. Arthritis Res Ther. 2012;14(2): R74.

16. Jay $G D$, Britt $D E$, Cha $C$. Lubricin is a product of megakaryocyte stimulating factor gene expression by human synovial fibroblasts. J Rheumatol. 2000; 27(3):594-600.

17. Jay GD, Waller KA. The biology of lubricin: near frictionless joint motion. Matrix Biol. 2014;39:17-24.

18. Waller KA, Zhang LX, Jay GD. Friction-induced mitochondrial dysregulation contributes to joint deterioration in Prg4 knockout mice. Int J Mol Sci. 2017; 18(6).pii:E1252.

19. Al-Sharif A, Jamal M, Zhang LX, Larson K, Schmidt TA, Elsaid KA. Lubricin/ proteoglycan 4 binding to CD44 receptor: a mechanism of the suppression of proinflammatory cytokine-induced synoviocyte proliferation by lubricin. Arthritis Rheumatol. 2015;67(6):1503-13.

20. Iqbal SM, Leonard C, Regmi SC, De Rantere D, Tailor P, Ren G, et al. Lubricin/Proteoglycan 4 binds and regulates the activity of Toll-like receptors in vitro. Sci Rep. 2016:6:18910.

21. Alquraini A, Garguilo S, D'Souza G, Zhang LX, Schmidt TA, Jay GD, et al. The interaction of lubricin/proteoglycan 4 (PRG4) with Toll-like receptors 2 and 4: an anti-inflammatory role of PRG4 in synovial fluid. Arthritis Res Ther. 2015;17:353.

22. Alquraini A, Jamal M, Zhang L, Schmidt T, Jay GD, Elsaid KA. The autocrine role of proteoglycan-4 (PRG4) in modulating osteoarthritic synoviocyte proliferation and expression of matrix degrading enzymes. Arthritis Res Ther. 2017;19(1):89.

23. Qadri M, Jay GD, Zhang LX, Richendrfer H, Schmidt TA, Elsaid KA. Proteoglycan-4 regulates fibroblast to myofibroblast transition and expression of fibrotic genes in the synovium. Arthritis Res Ther. 2020;22:113.

24. Qadri MM, Jay GD, Ostrom RS, Zhang LX, Elsaid KA. CAMP attenuates TGF$\beta^{\prime}$ s profibrotic responses in osteoarthritic synoviocytes: involvement of hyaluronan and PRG4. Am J Physiol Cell Physiol. 2018;315(3):C432-43.

25. Rhee DK, Marcelino J, Baker M, Gong Y, Smits P, Lefebvre V, et al. The secreted glycoprotein lubricin protects cartilage surfaces and inhibits synovial cell overgrowth. J Clin Invest. 2005:115(3):622-31.

26. Hill A, Waller KA, Cui Y, Allen JM, Smits P, Zhang LX, et al. Lubricin restoration in a mouse model of congenital deficiency. Arthritis Rheumatol. 2015;67(11):3070-81.

27. Jay GD, Fleming BC, Watkins BA, McHugh KA, Anderson SC, Zhang LX, et al. Prevention of cartilage degeneration and restoration of chondroprotection 
by lubricin tribosupplementation in the rat following anterior cruciate ligament transection. Arthritis Rheum. 2010;62(8):2382-91.

28. Waller KA, Chin KE, Jay GD, Zhang LX, Teeple E, McAllister S, et al. Intraarticular recombinant human proteoglycan-4 mitigates cartilage damage after destabilization of the medial meniscus in the Yucatan minipig. Am J Sports Med. 2017;45(7):1512-21.

29. Qadri M, Jay GD, Zhang LX, Wong W, Reginato AM, Sun C, et al. Recombinant human proteoglycan-4 reduces phagocytosis of urate crystals and downstream nuclear factor kappa B and inflammasome activation and production of cytokines and chemokines in human and murine macrophages. Arthritis Res Ther. 2018;20(1):192.

30. Guermazi A, Hayashi D, Roemer FW, Zhu Y, Niu J, Crema MD, et al. Synovitis in knee osteoarthritis assessed by contrast-enhanced magnetic resonance imaging (MRI) is associated with radiographic tibiofemoral osteoarthritis and MRI-detected widespread cartilage damage: the MOST study. J Rheumatol. 2014;41(3):501-8.

31. de Lange-Brokaar BJ, loan-Facsinay A, van Osch GJ, Zuurmond A-M, Schoones J, Toes REM, et al. Synovial inflammation, immune cells and their cytokines in osteoarthritis: a review. Osteoarthritis Cartilage. 2012;20(12): 1484-99.

32. Usher KM, Zhu S, Mavropalias G, Carrino JA, Zhao J, Xu J. Pathological mechanisms and therapeutic outlooks for arthrofibrosis. Bone Res. 2019;7:9.

33. Vogel DY, Glim JE, Stavenuiter AW, Breuer M, Heijnen P, Amor S, et al. Human macrophage polarization in vitro: maturation and activation methods compared. Immunobiology. 2014;219(9):695-703.

34. Wang $L X$, Zhang $S X, W u H J$, Rong XL, Guo J. M2b macrophage polarization and its roles in diseases. J Leukoc Biol. 2019;106(2):345-58.

35. Briken V, Mosser DM. Editorial: switching on arginase in $\mathrm{M} 2$ macrophages. J Leukoc Biol. 2011;90(5):839-41.

36. Qadri M, Almadani S, Jay GD, Elsaid KA. Role of CD44 in regulating TLR2 activation of human macrophages and downstream expression of proinflammatory cytokines. J Immunol. 2018;200(2):758-67.

37. Misharin AV, Cuda CM, Saber R, Turner JD, Gierut AK, Haines GK, et al. Nonclassical Ly6C-monocytes drive the development of inflammatory arthritis in mice. Cell Rep. 2014;9(2):591-604.

38. Zhang X, Goncalves R, Mosser D. The isolation and characterization of murine macrophages. Curr. Protoc. Immunol. 2008;14:14.1.

39. Livak KJ, Schmittgen TD. Analysis of relative gene expression data using real-time quantitative PCR and the 2(-Delta Delta C(T)) method. Methods. 2001:25:402-8

40. Samson ML, Morrison S, Masala N, Sullivan BD, Sullivan DA, Sheardown $H$, et al. Characterization of full-length recombinant human proteoglycan 4 as an ocular surface boundary lubricant. Exp Eye Res. 2014;127C:14-9.

41. Quero L, Hanser E, Manigold T, Tiaden AN, Kyburz D. TLR2 stimulation impairs anti-inflammatory activity of M2-like macrophages, generating a chimeric M1/M2 phenotype. Arthritis Res. Ther. 2017;19:245.

42. Li JY, Liu Y, Gao XX, Gao X, Cai H. TLR2 and TLR4 signaling pathways are required for recombinant Brucella abortus BCSP31-induced cytokine production, functional upregulation of mouse macrophages, and the Th1 immune response in vivo and in vitro. Cell Mol Immunol. 2014;11(5):477-94.

43. Lech $\mathrm{M}$, Anders HJ. Macrophages and fibrosis: how resident and infiltrating mononuclear phagocytes orchestrate all phases of tissue injury and repair. Biochem Biophys Acta. 2013;1832(7):989-9.

44. Kurowska-Stolarska M, Alivernini S. Synovial tissue macrophages: friend or foe? RMD Open. 2017:3(2):e000527.

45. van Roojien N, Hendrikx E. Liposomes for specific depletion of macrophages from organs and tissues. Methods Mol Biol. 2010;605:189-203.

46. Blom $\mathrm{AB}$, van lent $\mathrm{PL}$, Libregts $\mathrm{S}$, Holthuysen $\mathrm{AE}$, van der Kraan $\mathrm{PM}$, van Roojien $\mathrm{N}$, et al. Crucial role of macrophages in matrix metalloproteinasemediated cartilage destruction during experimental osteoarthritis: involvement of matrix metalloproteinase 3. Arthritis Rheum. 2007;56(1):147157.

47. Sun AR, Wu X, Liu B, Chen Y, Armitage CW, Kollipara A, et al. Pro-resolving lipid mediator ameliorates obesity induced osteoarthritis by regulating synovial macrophage polarization. Sci Rep. 2019;9:426.

48. Scanzello CR. Chemokines and inflammation in osteoarthritis: insights from patients and animal models. J Orthop Res. 2017;35(4):735-9.

49. Zhu X, Lee CW, Xu H, Wang Y-F, Yung PS, Jiang Y, et al. Phenotypic alteration of macrophages during osteoarthritis: $\mathbf{s}$ systematic review. Arthritis Res Ther. 2021:23:110.
50. Wang W, Lin X, Xu H, Sun W, Bouta EM, Zuscik MJ, et al. Attenuated joint tissue damage associated with improved synovial lymphatic function following treatment with bortezomib in a mouse model of experimental posttraumatic osteoarthritis. Arthritis Rheumatol. 2019:71(2):244-57.

51. Sambamurthy N, Zhou C, Nguyen V, Smalley R, Hankenson KD, Dodge GR, et al. Deficiency of the pattern-recognition receptor CD14 protects against joint pathology and functional decline in a murine model of osteoarthritis. PLoS One. 2018;13(1):e0206217.

\section{Publisher's Note}

Springer Nature remains neutral with regard to jurisdictional claims in published maps and institutional affiliations.

\section{Ready to submit your research? Choose BMC and benefit from:}

- fast, convenient online submission

- thorough peer review by experienced researchers in your field

- rapid publication on acceptance

- support for research data, including large and complex data types

- gold Open Access which fosters wider collaboration and increased citations

- maximum visibility for your research: over $100 \mathrm{M}$ website views per year

At BMC, research is always in progress.

Learn more biomedcentral.com/submissions 